\title{
Challenges and Opportunities of Internet of Things in Healthcare
}

\author{
Amine Rghioui, Abdelmajid Oumnad \\ Electronic and Communication Laboratory-LEC, EMI, Mohammed V University in Rabat, Morocco
}

\begin{tabular}{|c|c|}
\hline Article Info & ABSTRACT \\
\hline Article history: & The Internet of Things (IoT) relies on physical objects interconnected \\
\hline Received Sen $12 \quad 2017$ & ch other's, creating a mesh of devices producing information and \\
\hline Received Sep 12, 2017 & services. In this context, sensors and actuators are being continuously \\
\hline Revised Dec 22, 2017 & embedded in everyday objects (e.g., cars, home appliances, and smartphones) \\
\hline Accepted Jun 12, 2018 & $\begin{array}{l}\text { thus pervading our living environment. Among the plethora of application } \\
\text { contexts, smart Healthcare is gaining momentum. Indeed IoT can }\end{array}$ \\
\hline Keyword: & $\begin{array}{l}\text { revolutionize the healthcare industry by improving operational efficiency and } \\
\text { clinical trials' quality of monitoring, and by optimizing healthcare costs. This }\end{array}$ \\
\hline $\begin{array}{l}\text { Big data } \\
\text { Embedded systems } \\
\text { Healthcare }\end{array}$ & $\begin{array}{l}\text { paper provides an overview of IoT, its applicability in healthcare, some } \\
\text { insights about current trends and an outlook on future developments of } \\
\text { healthcare systems. }\end{array}$ \\
\hline
\end{tabular}

Copyright $@ 2018$ Institute of Advanced Engineering and Science.
All rights reserved.
Internet of Things
Copyright (c) 2018 Institute of Advanced Engineering and Science.
All rights reserved.

\section{Corresponding Author:}

Amine Rghioui,

Electronic and Communication Laboratory-LEC,

EMI, Mohammed V University in Rabat, Morocco.

Email: rghioui.amine@gmail.com.

\section{INTRODUCTION}

The Internet of Things (IoT) [1] is a computing concept that describes a future where every day physical objects will be connected to the Internet and be able to identify themselves and interact locally or remotely with other devices, IoT is expanding rapidly (by 2020 there will be more than 50 billion IoT devices on the Internet [2]), and it is transforming every application domain, including healthcare by enabling the introduction of smart services that help improving health and well-being, increase public safety, and enable better shopping experience. Such smart services lead to better quality of living, and they are currently and extensively fueling the development of Healthcare domain under the hood, these smart services manage also a lots of data coming both from conventional computing systems and novel IoT devices, such as wearables, sensor networks, etc. To properly manage them, and contemporarily tackle the enormous data availability registered over the last two decades.

Over the last two decades, big data has been widely recognized as an effective computing paradigm, gaining consensus from universities, governments, and enterprises [3].

This paper presents a review of the IoT technologies and their applications in Healthcare domain and indicates also some interesting research areas. In detail, Section II presents and explaining other surveys on IoT in Health care. Section III contains revolution of Internet of Things technologies. Application of IoT in healthcare are discussed in Section IV, and section V explains architecture and protocol of IoT in Healthcare. Finally the conclusion.

\section{RELATED WORKS}

Internet of Thing can change style life of human [4]. Recently there have been a number of independent researches that investigated the potential of integrating concepts of the IoT in Healthcare 
domain. IoT can provide several benefits to improve the quality of life for citizens and prove lifestyle suggestions for welfare. To clarify that the IoT refers to several good surveys recently presented each display security challenges in Healthcare. There are several documents published survey covering different aspects of IoT technology in Healthcare. For example, the survey by Riazul Islam [5] covers the main communication enabling technologies, wired and wireless, and the elements of wireless sensor networks (WSN). Among these investigations, Jaime Lioret and her colleagues [6] presented a Smart Communication Architecture for Ambient Assisted Living. Another recent study, inspired by Yujun Ma [7], presented Big Health Application System based on Health Internet on Things and Big Data.

To the best of our knowledge, however, no investigation has focused on industrial solutions of IoT. All the above investigations have reviewed the solutions proposed by academic and research communities and to refer to scholarly publications produced by the respective researchers. In this article, we review the problems, challenges, technologies and IoT applications in Healthcare that are proposed, designed, developed and marketed, and are useful for researchers and industrial organizations.

The contour of the contributions of this paper compared to the recent literature in the field can summarized as follows:

a. Compared to other documents from the field survey, this survey provides a deeper summary of the Internet of Things in Healthcare, which allows us to know what the Internet of Things is in details.

b. We explore the relationship between the IoT and other emerging technologies: sensor networks, RFID technology, and cloud computing.

c. We express the need for better horizontal integration between IoT services.

d. We also present the different fields of application of the Internet of Things in Healthcare and the human life.

\section{IOT TECHNOLOGIES}

\subsection{Radio Frequency Identification (RFID)}

Radio Frequency Identification (RFID) is an automatic and contactless technology, providing a communication interface with the tagged objects through wireless data transmission to retrieve relevant information [8]. RFID allows automatic identification and data capture using radio waves, a tag, and a reader. The tag cab store more data than traditional barcodes. Three types of tags are used.

Table 1. Types of RFID tagsServices and benefits in the field of smart cities

\begin{tabular}{cl}
\hline Passive RFID tags & They are not battery-powered. Tag reading is quick (a reader can read up to 250 tags per second) \\
\hline Avtive RFID tags & Are used in manufacturing, hospital laboratories, and remote-sensing \\
Semi-Passive RFID tags & Use batteries to power the microchip while communicating by drawing power from the reader. \\
\hline
\end{tabular}

In the IoT scenario, a key role is played by RFID systems, composed of one or more readers and several tags. These technologies help in automatic identification of anything they are attached to, and allow objects to be assigned unique digital identities, to be integrated into a network, and to be associated with digital information and services.

\subsection{Near Field Communication (NFC)}

It is similar to RFID configuration. Near Field Communication (NFC) can be made customeroriented by integration of RFID reader into mobile phones. In addition, it is type of radio communication between NFC mobile devices by connecting them together in the domain of other phone. Wireless Sensors Network (WSN). The NFC technology can play a significant role in the future progress of IoT. It will enable to provide necessary tool to be wirelessly connected to other smart objects [9]. For example by using NFC mobile a user will be able to transfer the mobile set into other various objects like mobile set will be able to used as a credit card.

\subsection{Machine-to-Machine Communication (M2M)}

Machine-to-Machine Communication (M2M) refers to the communications between computers, embedded processors, smart sensors, actuators and mobile devices. This sort of communication is increasing these days. There are four components of M2M, that are sensing, heterogeneous access, information processing and applications \& processing. It has applications in different sectors like healthcare, smart robots, cyber transportation systems (CTS), manufacturing systems, smart home technologies, and smart grids [10]. 


\subsection{Wireless sensor networks (WSN)}

A Wireless Sensor Network (WSN) can be defined as a network of small embedded devices, called sensors, which communicate wirelessly following an ad hoc configuration. WSNs consist of spatially distributed autonomous sensor-equipped devices to monitor physical or environmental conditions and can cooperate with RFID systems to better track the status of things such as movements, pressure, temperature, and location.

WSNs may provide various useful data and are being utilized in several areas like healthcare, government and environmental services (natural disaster relief), defence (military target tracking and surveillance), hazardous environment exploration, seismic sensing. For example, General Electric deployed sensors in its jet engines, turbines, and wind farms. By analysing data in real time, General Electric saves time and money associated with preventive maintenance. Likewise, American Airlines uses sensors capable of capturing 30 terabytes of data per flight for services such as preventive maintenance.

\subsection{Big Data}

The Big Data is a recent term associated with the huge amounts of stored /obtained data due to the revolutionary advances in different technologies including: cloud computing, spread of social media, and wireless communication technologies. It is defined according to the size of data (volume), types of data based on the producing source (variety), and the time frequency to generate the data (velocity); every, minute, day, month, or a decade.

\section{INTERNET OF THINGS IN HEALTHCARE}

The rising costs of health care and the increasing availability of new personal health devices are the ingredients of the vision of the Internet of Things in the connected healthcare. The vision of connected healthcare is growing because of the availability of new technological tools [11]. By the application of the IoT and new technologies, it is possible to create a health application that appears every morning to request reading the level of glucose in the blood and collects data from the patient automatically. In the vision of connected healthcare, patients are those who take control of their health and being in good physical and mental health due to this application. In addition, this leads to a good responsibility and control of heath by allowing a real scenario for IoT in care health. [12]. IoT will help doctors to respond quickly in emergencies and allow them to cooperate with international hospitals to track the status of a patient. There are also other applications of IoT such as patient identification; this application aims to reduce adverse events for patients, maintenance of comprehensive electronic medical records. [13].

\subsection{Challenges of Healthcare}

Most regions of the world face a great challenge to manage child mortality, aging population, and difficult living environments with poor health. Although the global demand for medical services is increasing in recent years, in which citizens visit doctors when they get sick. We citizens briefly some of the challenges of the Internet of Things in Healthcare:

Adherence monitoring: Physicians do not have the means to better assess whether their patients are following prescribed treatment that may include medication, rehabilitation exercises, and preventive activities such as diet avoidance. It is quite common that lack of adhesion increases the risk of hospitalization and, as a result, increases the economic burden for patients and their families.

Limited and prospective time: the increase in the population results in increased disease and disability limits doctors to check each patient with quality time. Because of the short time of screening, physicians lack the daily routine of the patient, such as physical activity, diet, sleep and social life, all these attributes are equally important in the diagnosis and treatment process.

Integration of multiple devices and protocols: The diversity of devices involved in networks is another challenge for the success of IoT in health care. Ensuring that multiple devices are connected to each other and that multiple users communicate effectively with each other is a problem. The difficulty lies in the fact that many device manufacturers do not have an agreed set of communication protocols and standards. Although a variety of mobile devices can be connected to the network and actively collect data, different communication protocols complicate the aggregation process. [14].

Another challenge is the security challenge, which involves managing credentials and controlling access to patient requests and confidential information. For example, health care providers are allowed access to devices in response to demand from the patient's sensor devices, but the Internet connection used may be a public or unstable Wi-Fi network that can easily be man-in -the-middle. Many authentication techniques could be put in place so that patients are able to verify and allow physicians to access their internal devices. 


\subsection{Benefits to Patient}

Researchers and analysts say that the Internet of things is one of the most sophisticated technologies that can improve the health, productivity and security of billions of people. Here are the advantages:

a. Improved quality of life: Awareness of children's health and a new educational culture based on the needs of children with health problems. This has motivated health researchers to develop a special IOT service called Child Health Information (CHI) to address this need effectively. In this regard, an interactive tutorial in a paediatric ward offering CHI services to educate, amuse and empower in-hospital children is proposed in [15], and an online IoT health service that can encourage children To acquire good nutritional habits.

b. Blood glucose monitoring reveals individual patterns of blood glucose changes and helps to plan meals, activities and drug times. Some researcher proposes an m-IoT configuration method for non-invasive glucose detection in real time. In this method, patient sensors are linked by IPv6 connectivity to the healthcare providers involved. The utility model in [16] discloses a transmission device for the transmission of somatic data collected on blood glucose from IoT networks. This device includes a blood glucose meter, a mobile phone or a computer, and a processor in the background. There is also a generic medical acquisition detector based on the Internet of Things that can be used to monitor glucose levels in diabetes:

1) Disease management in real time:

2) Improved User Experience:

3) Reduced cost of care:

4) Improved Patient outcome:

5) Improved quality of life:

6) Time saving:

\section{IOT ARCHITECTURE IN HEALTHCARE}

The IoT should be able to interconnect billions or trillions of heterogeneous objects across the Internet, so there is a critical need for a flexible architecture layers. The growing number of available architectures has not converged to a reference model. In the variety of models on offer, the base model is a 3layer architecture consisting in the application, network, and Perception layers. The 3-layer structure is composed of Perception layer, the network layer and the application layer, as shown in Figure 1.[17].

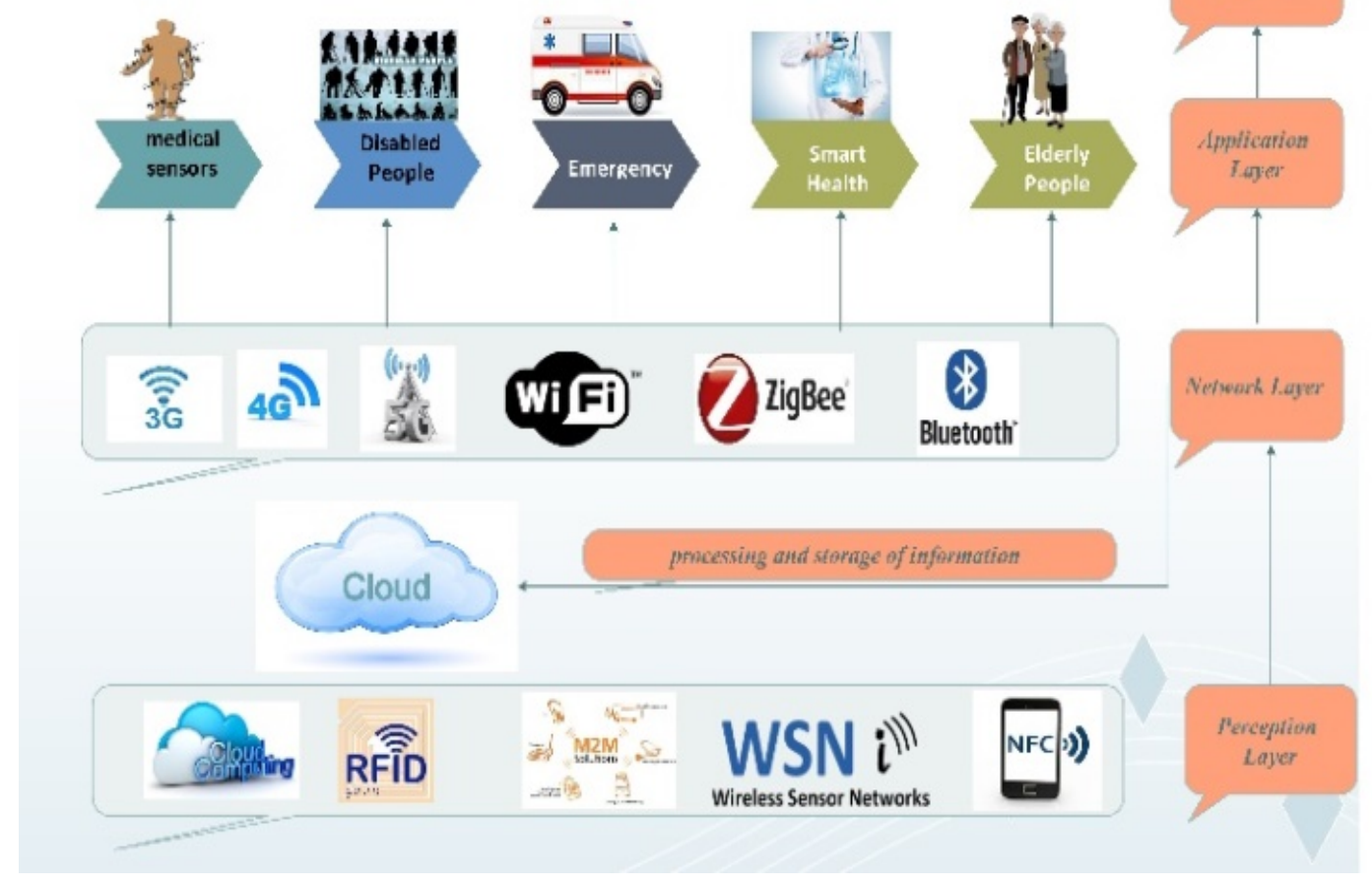

Figure 1. IoT reference architecture in healthcare 


\subsection{The Perception Layer}

This layer focuses on the identification of objects and collecting information. It is also known as "layer system". It composed of the physical objects and detection devices. Perception layer comprises terminals and sensor networks. The sensors may be RFID, bar code or 2D-infrared sensors depending on the object identification method. Depending on the type of sensors, information can be on location, temperature, direction, movement, vibration, acceleration, humidity, chemical changes in the air, etc. The collected information is transmitted to the network layer for transmission.

\subsection{The Network Layer}

The main function of network layer is to transfer the information collected from Device layer to Service layer. The middle layer consists of a network of interconnected smart gateways. This model enables developers and end-users to exploit cloud services with a minimum knowledge of the underlying hardware and infrastructure. The network layer has the competence to improve the exploitation of information on the network. In addition, the network layer is able to aggregate information from existing IT infrastructures (e.g. business systems, transportation systems, power systems, health care systems, etc.).

\subsection{Application layer}

The main task of the application layer is to find out the service. The application layer is a connecting IoT technology, a layer for achieving broad intelligent application providing various solutions. For the application layer the key issue is to share information on communities and ensure information security.

\section{HEALTHCARE PLATFORM}

Many researchers have worked on designing and implementing various IoT-based healthcare services and on solving various technological and architectural problems. In this section, we showed the main research areas considered by most platforms the field of the Internet of Things Healthcare. BodyCloud is a software architecture (SaaS) that supports the storage and management of data flows of body sensors and the processing of data stored using software services hosted in Cloud. BodyCloud strives to support multiple interdisciplinary applications and specialized processing tasks, enabling large-scale data sharing and collaborations between users and applications in Cloud, and providing cloud services via sensor-rich mobile devices. Bodycloud has been used to support research prototypes in healthcare fields, and provides decision support services to take other actions based on the data analysed. [18]

Kaa, is an open-source IoT platform, allows healthcare system integrators to establish cross-device connectivity and implement smart features into medical devices and related software systems. Healthcare companies-developing hardware or software products-that have chosen to integrate Kaa's functionality into their products were able to achieve IoT goals faster and at little expense. By utilizing the Kaa platforms, a finished product for clinical telemedicine and remote patient monitoring can be functional in a matter of weeks. Kaa can be integrated with portable or stationary medical equipment; it can even fit into a tiny sensor. Once your device is connected Kaa will begin to monitor and collect any type of data generated by the device. The Kaa feature set addresses a broad range of smart hospital functions such as connected rooms, smart alerts and notifications, and electronic medical record management [19].

Majid A. Al-Taee and his colleagues Presents a new platform based on the Internet of Thing (IoT) to support the self-management of diabetes. This mobile health approach (mHealth) enables multiple diabetes care dimensions by means of remote collection and tracking of patient data and personalized on a smartphone platform. Such support for self-management of diabetes allows for real-time clinical interaction and feedback tailored to the patient's personal needs, using current and historical patient data. The platform compares the patient's measurements and activities with the standards given by the physician and generates appropriate warnings and advice in terms of feedback notices. [20].

Boyi $\mathrm{Xu}$ and his colleagues in [21] developed a three-layer cloud platform to access omnipresent data on the cloud via IoT Healthcare. The tenant database layer stores multi-tenant databases. The resource layer is responsible for controlling data access, and the business layer performs the coordination required for data sharing and interoperability. It should be noted that distributed health data is organized using a resource control mechanism.

\section{IOT APPLICATION IN HEALTHCARE}

In this part, we introduce several applications that are designed to give support to human and especially in the fields of health; we can classify them in two groups, general applications, and clinical applications. 


\subsection{Healthcare}

The rising costs of health care and the increasing availability of new personal health devices are the ingredients of the vision of the Internet of Things in the connected healthcare. The vision of connected healthcare is growing because of the availability of new technological tools. By the application of the IoT and new technologies, it is possible to create a health app that appears every morning to request reading the level of glucose in the blood and collects data from the patient automatically. In the vision of connected healthcare, patients are those who take control of their health and being in good physical and mental health due to this application. In addition, this leads to a good responsibility and control of heath by allowing a real scenario for the Internet of Things (IoT) in care health.

IoT will help doctors to respond quickly in emergencies and allow them to cooperate with international hospitals to track the status of a patient. The IoT applications can be found also in home monitoring especially for elderly people with special needs or chronic illnesses such as diabetes, congestive heart failure.... There are also other applications of IoT such as patient identification; this application aims to reduce adverse events for patients, maintenance of comprehensive electronic medical records. [22]. Internet of Things based on RFID tags in the medical domain enable rapid and accurate identification of every intelligent entity, enabling a universal and fast access to personal health records on an Internet of Things.

\subsection{People with disabilities}

People who are hearing impaired can benefit from external or internal devices (implanted in the ear) to improve hearing. On the other hand, a wireless low cost glove designed to help the deaf communicate with those who are not familiar. It recognizes hand signals and converts them into voice interface with a Java-enabled monitoring station (mobile phone). The glove is equipped with bending sensors (passive resistive devices that may be used to detect the bending or flexion) along the fingers. In another scenario, people with visual impairments independent shop.

The blind navigation system helps them find their way into a store. The RFID system of the store can use software to guide the visually impaired in shopping. The supermarket is divided into cells containing a shelf and cells pass. RFID tags are distributed across the floor. Module monitoring station (smartphone) allows the person with a visual impairment to say the grocery section he/she wants to go. The IoT is also used to monitor and control various components in cars. Ford and Intel have teamed up in 2014 to explore new possibilities to customize the user experience by using facial recognition software and an application of mobile phone. To provide better privacy controls and identify the various drivers and automatically adjust the characteristics according to the preferences of an individual [23].

\subsection{Tracking and Monitoring of objects and persons}

Monitoring is the function aims at the identification of a person or an object in motion [26]. In the field of health there are tracking and monitoring of patient flow to improve the workflow in hospitals and motion tracking through choke points, such as access to designated areas. The follow-up is more frequently applied instead of permanent inventory of monitoring (e.g. for maintenance, availability in case of need and use monitoring) and tracking of materials in order to avoid problems during surgery.

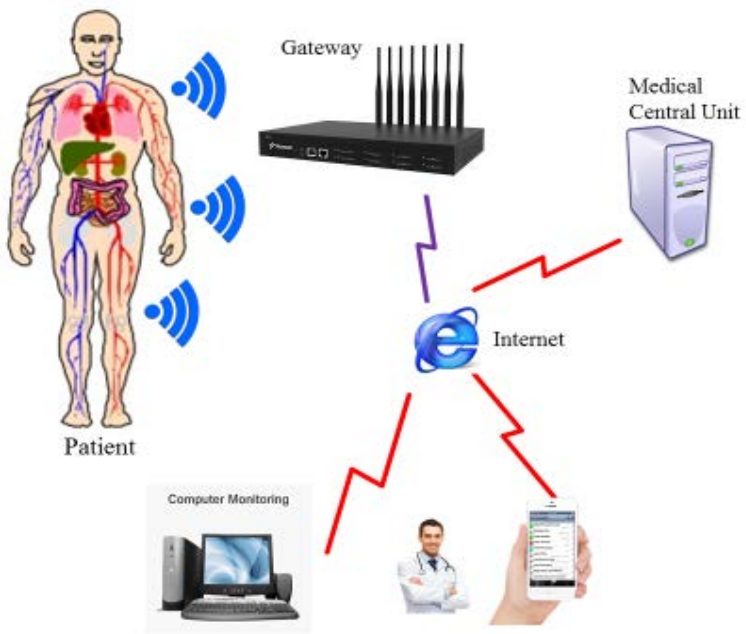

Figure 2. IoT Healthcare monitoring System 


\subsection{Identification and Authentification}

The identification of the patients, is to reduce malicious patient incidents (e.g. bad drug/dose/time/procedure), also the identification of the medical file complete and current, with regard to staff, the identification and authentication is most often used to grant access and improve the morale of the employees based on the safety of patients, are used as identification and authentication to meet the requirements of safety procedures to prevent the theft or loss of products and instruments [24].

\subsection{Transport and Data Collection}

Nowadays, personal health devices can transmit data using short-range Bluetooth wireless technologies, Near Field Communication (NFC), ZigBee or Bluetooth Low Energy (BLE). Transfer and automatic data collection is to reduce the time of processing forms, automated care and audit and management of medical procedures. This function also refers to integration RFID technology.

\subsection{Clinical Care}

Sensors will help hospitalized patients to move freely within the hospital without having to commit to a certain rooms and been liked wired to certain machines, as well as avoiding the trouble moving from a pavilion to the other for examination and analysis. It will help as well as caregivers in the performance of their work, as will enable it to track the patient's condition remotely and help them to cooperate with each other in the diagnosis of the patient's condition between the various disciplines. Moreover, it will save the doctors' time from moving between patients to reveal their health status. It will help them for rapid intervention in an emergency and will enable them to cooperate with international hospitals to track a patient's condition.

\subsection{Continuous Cardiac Monitoring}

A Continuous Surveillance Unit (USC) is a service of the hospital, designed to receive and care for patients in need of close supervision. The looming health crisis attracts researchers and industry to look for optimal and rapid solution to perform cardiac remote monitoring, with medical records updates in real-time via the Internet, by economic solutions valid for the entire world.

A wearable WBAN is efficient for this mission [25]. It can be placed on specific places of the body of the patient to continuous measure its Electrocardiograms signs (ECG) and transmit them to the hospital supervisor medical central unit in real-time. Thus, it can monitor patient under natural physiological states of health in the long term without constraining their normal activities. These sensors are able to gather information from Implantable Cardiac Defibrillators (ICDs) to detect and treat ventricular tachyarrhythmia and prevent Sudden Cardiac Death (SCD) as shown in Figure 3.

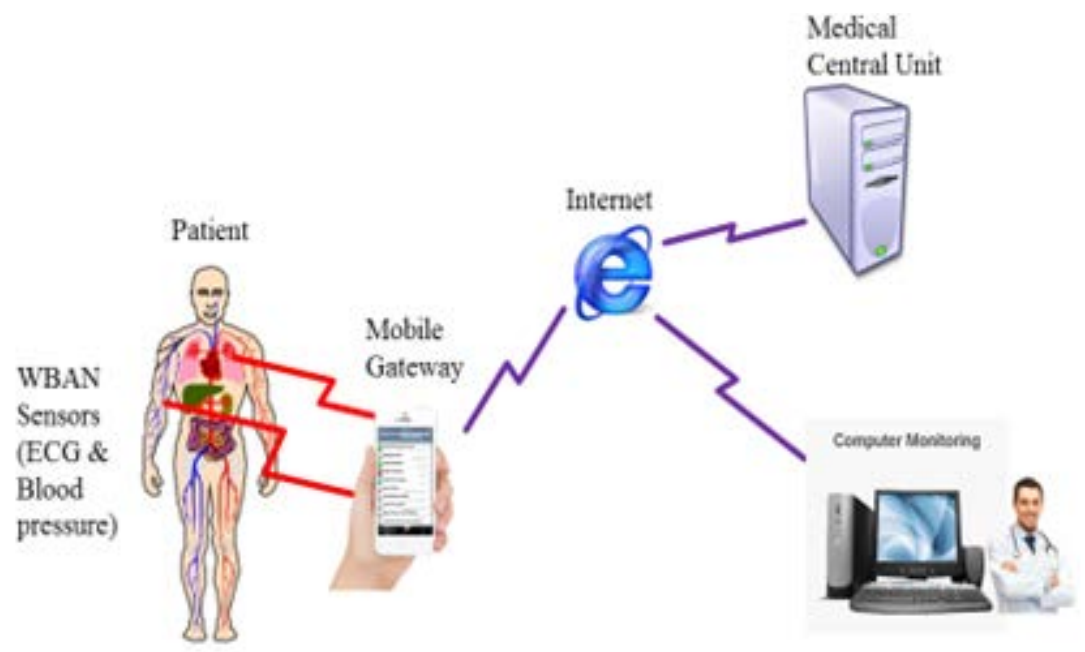

Figure 3. Example of IoT based healthcare system

\section{CONCLUSION}

The healthcare system today is on a trajectory that is unsustainable. The bulk of costs in the current system are due to patients having chronic care diseases. Thus, a focus in the future should be on preventive care as well as population health management and overall wellness. With Internet of Things, health 
management of a population can be understood better. Here it should be summarized the paper contribution. Future work will consist in comparing and evaluating algorithms and techniques used in reality healthcare-related data mining and in the implementation of IoT solutions in healthcare systems.

\section{REFERENCES}

[1] Coetzee.L, Fksteen.J, “The internet of things - promise for the future? An introduction”, IST-Africa Conference Proceedings, 2011.

[2] M. Aazam, I. Khan, A. A. Alsaffar, E.-N.Huh, "Cloud of internet integrating Internet of things and cloud computing and the issues involved”, 11th International Conference on Applied Sciences and Technology (IBCAST), 2014.

[3] Luigi Atzori, Antonio Iera, Giacomo Morabito, “The Internet of Things: A survey”, Computer Networks, vol.54, pp. 2787-2805, 2010.

[4] A. Rghioui, A. Oumnad, "Internet of Things: Surveys for Measuring Human Activities from Everywhere", International Journal of Electrical and Computer Engineering (IJECE), vol/issue: 7(5), pp. 2474-2482, 2017.

[5] S. M. Riazal Islam, D. Kwak, MD. Humaun Kabir, M. Hossain, K. Kwak, "The Internet of Things for Health Care: A Comprehensive Survey”, IEEE Access, vol.3, pp: 678-708, 2015

[6] J. Lioret, A. Canovas, S. Sendra, L. Parra, “ A smart communication architecture for ambient assisted living”, IEEE Communication Magazine, vol.53, pp: 26-33, 2015

[7] Y. Ma, Y. Wang, J. Yang, Y. Miao, W. Li, Big HealthApplication System based on Health Internet of things and Big Data”, IEEE Access, 2016

[8] I. Erguler, “A potential weakness in RFID-based Internet-of-Things systems”, Pervasive and Mobile Computing, vol. 20, pp: 115-126, 2015.

[9] S. Karpischek, F. Michahelles, F. Resatsch, E. Fleisch, "Mobile sales assistant - an NFC-based product information system for retailers", Proceedings of the First International Workshop on Near Field Communications, 2009

[10] M. Ptick, V. Čačković; M. Pavelić; M. Kušek; G. Ježić, “ Architecture and Funcionality in M2M Standards”, 2015 38th International Convention on Information and Communication Technology, Electronics and Microelectronics (MIPRO), pp: 413-418, 2015

[11] Danilo F.S. Santos, Hyggo O. Almeida, Angelo Perkusich, “A personal connected health system for the Internet of Things based on the Constrained Application Protocol”, Computers and Electrical Engineering, vol 15, 2015

[12] A. Santos, J. Macedo, A. Costa, M. J. Nicolau, «Internet of Things and Smart Objects for M-Health Monitoring and Control », Procedia Technology, vol.16, pp : 1351 - 1360, 2014

[13] K. Kai, P. Zhi-bo, W. Cong, « Security and privacy mechanism for health internet of things », The Journal of China Universities of Posts and Telecommunications, Vol. 20, pp : 64-68, December 2013.

[14] http:// blog.intrepid.io/iot-in-healthcare-challenges

[15] S.Vicini, S. Bellini, A. Rosi, and S. Sanna, “An Internet of Things enabled interactive totem for children in a living lab setting,” in Proc. ICE Int. Conf. Eng., Technol. Innov. (ICE), Jun. 2012, pp. 1-10.

[16] Z. J. Guan, "Somatic data blood glucose collection transmission device for Internet of Things," Chinese Patent 202838653 U, Mar. 27, 2013.

[17] EU FP7 Internet of Things Architecture project? 2014 Available: http://www.iot-a.eu/public.

[18] P. Pace, G. Aloi, R. Gravina, G. Fortino, G. Larini, M. Gulino “Towards Interoperability of IoT-based Health care platforms: the INTER-Health use case”, 11th EAI International Conference on Body Area Networks, pp: 12-18, 2016.

[19] http:// www.kaaproject.org/healthcare/

[20] M. A. Al-Taee, W. Al-Nuaimy, A. Al-Ataby, Z. J. Muhsin,S. N. Aboud, "Mobile health platform for Diabetes management based on the Internet of Things”, IEEE Jordan Conference on Applied Electrical Engineering and Computiong Technologies (AEECT), pp: 1-5, 2015

[21] B. Xu, L. D. Xu, H. Cai, C. Xie, J. Hu, and F. Bu, "Ubiquitous data accessing method in IoT-based information system for emergency medical services,’’ IEEE Trans. Ind. Informat., vol. 10, no. 2, pp. 1578-1586, May 2014.

[22] A. Rghioui, S. Sendra, J. Lloret, A. Oumnad, "Internet of Things for Measuring Human Activities in Ambient Assisted Living and e-Health”, Network Protocols and Algorithms, vol.8, no. 3, 2016, pp. 15-28.

[23] M. C. Domingo, "An overview of the Internet of Things for people with disabilities », Journal of Network and Computer Applications, vol. 35, pp: 584-596, 2012.

[24] M. Anwar, A. H. Abdullah, K. N. Qureshi, A. H. Majid, "Wireless Body Area Networks for Healthcare Applications: An Overview”, TELKOMNIKA Telecommunication, Computing, Electronics and Control., vol. 15, no. 3, pp. 1088-1095, 2017

[25] A. Santos, J. Macedo, António Costa, M. JoãoNicolau, "Internet of Things and Smart Objects for M-Health Monitoring and Control”, Procedia Technology, vol.16, pp: 1351-1360, 2014.

[26] H. S. Kim, et al., "A Daily Activity Monitoring System for Internet of Things-Assisted Living in Home Area Networks,” International Journal of Electrical and Computer Engineering (IJECE), vol/issue: 6(1), pp. 399-405. 


\section{BIOGRAPHIES OF AUTHORS}

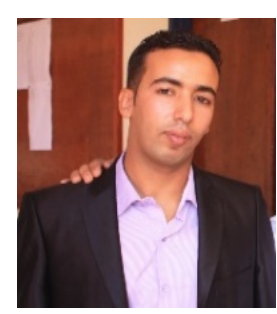

Amine RGHIOUI was born in Morocco, in 1989. He received the B.S. degree from Faculty of Sciences, Fez, Morocco in 2012 and M.S from Faculty of Sciences, Kenitra, Morocco in 2014.

He is currently member of Electronic and Communication laboratory at Mohammadia School of Engineering. His research interests include wireless sensor network, Internet of Things and connected objects, Big Data.

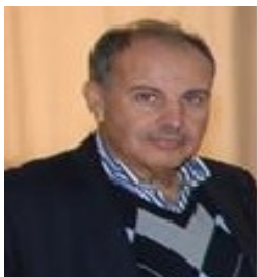

Dr. Abdelmajid OUMNAD Professor in the Department of Electrical Engineering at Mohammadia School of engineering (EMI) and member of the Laboratory of Electronics and Communication (EMI). He received his Ph.D. in electronics of the Claude Bernard University in Lyon, He is a professor in Electronic and Communication Technology at the Department of Electrical Engineering in Mohammadia School of engineering in Rabat. 